\title{
Blocking innate immunity to slow the progression of chronic kidney disease
}

\author{
Christoph Schmaderer • Uwe Heemann
}

Received: 29 July 2014 / Accepted: 30 July 2014 / Published online: 10 August 2014

(C) Springer-Verlag Berlin Heidelberg 2014

Chronic inflammation is present in almost every biopsy of patients with chronic kidney disease. It is independent of the cause of kidney injury and can be detected in acute kidney failure and chronic kidney allograft nephropathy, autoimmune diseases like lupus nephritis or granulomatosis with polyangiitis, and even diabetic nephropathy. Chronic inflammation does not seem to be triggered by pathogens be it viruses, bacteria, or bacterial products (Leemans et al. 2014).

During the last few years, numerous endogenous ligands have been identified that can bind to damage-associated molecular patterns (DAMPs) and trigger inflammation by activating nuclear factor kappa $b$ and secreting cytokines and chemokines (Leemans et al. 2014). The resulting kidney tissue damage is associated with the release of more endogenous ligands and, therefore, ends in a self-sustaining vicious circle. The idea of blocking a crucial molecule in the innate immunity pathway namely interleukin-1 receptor-associated kinase 4 (IRAK-4) is, therefore, a reasonable attempt to ameliorate kidney damage.

In this issue of Naunyn-Schmiedeberg's Archives of Pharmacology, Kondo et al. report their experience with the use of a novel IRAK-4 inhibitor in a rat model of 5/6 nephrectomy (Kondo et al. 2014). In their remnant kidney model, ablation of one kidney to reduce renal mass is followed by unilateral nephrectomy. Consecutively, the rat develops clinical signs of chronic kidney disease (CKD) with rising creatinine, development of hypertension, and proteinuria (Kliem et al. 1996). Along with this clinical deterioration of kidney function, glomerular scarring, tubular atrophy, fibrosis, and infiltration of immune cells are observed. Similar models have

C. Schmaderer $(\bowtie) \cdot$ U. Heemann

Department of Nephrology, Klinikum rechts der Isar, Technische

Universität München, Ismaninger Straße 22, 81675 Munich,

Germany

e-mail: christoph.schmaderer@1rz.tu-muenchen.de been used extensively to understand the progression of chronic kidney disease and to test the influence of pharmacological intervention on its course.

Kondo et al. (2014) use a novel IRAK-4 inhibitor in this model. The inhibitor blocks downstream pathways of the Tolllike receptors (TLRs) and IL-1 and IL-18 receptors and, therefore, interferes significantly with the innate immune system. Because IRAK-4 is the only molecule of the IRAK family that is indispensable for this pathway, it is a very promising target to block inflammation not only in the kidney as the TLR pathway is present in nearly every cell of the body (Wang et al. 2009).

\section{Is AS2444697 able to significantly block inflammation?}

In a sepsis model with intraperitoneal injection of LPS AS2444697, Kondo et al. (2014) report a significant reduction of systemic inflammatory markers that is clearly dose-dependent. Unfortunately, there is no experimental group that examined overall survival. In conclusion, we can notice that AS2444697 is able to reduce systemic inflammation after administration of an exogenous TLR 4 ligand.

\section{Is AS2444697 able to ameliorate chronic progressive kidney disease in a remnant kidney model?}

Looking at the creatinine clearance, there is a significant amelioration of excretory kidney function, at least in the high-dose group. Considering the rather low size of the experimental groups with six to eight animals, one might even think the effect could be present also in the low-dose group. Kidney weight was lower in the AS2444697-treated animals which might be due to less fibrosis which so far is only speculative as there is no quantitative measurement of, for 
example, hydroxyprolin content because evaluation of fibrosis in light microscopic slides is rather subjective and can just give us a hint whether there are huge effects. The same is true for all other histological parameters. Especially in interstitial cell infiltration, we would consider quantification by staining with a pan-leukocyte marker as gold standard. Of particular interest would be a quantification of the immunophenotype of infiltrating cells in the remnant kidney as this would give precious information on the mechanism of amelioration of kidney function.

Systemically administered AS2444697 will most likely block IRAK-4 in every cell, shutting down a crucial TLR pathway. Thus, the effects observed might be caused by a reduced infiltration of cells that are functionally deficient of TLR/IL1R pathways and consecutively reduce tissue damage and the release of endogenous TLR ligands. On the other hand, tubular cells and other kidney cell structures also show a significant amount of TLR/IL1 receptors, and AS2444697 will also block inflammation in these cells.

\section{The role of inflammation and fibrosis in chronic kidney disease}

Kidney slides derived from patients with chronic renal insufficiency always reveal the same findings: tubular atrophy, tubular dilation, interstitial fibrosis, glomerular scaring, arteriolar wall thickening, and infiltration of inflammatory cells in the interstitial space. Based on these histological features and taking into account the possible pathophysiology, pharmacological interventions were primarily based on blood pressure lowering and the reduction of glomerular filtration with the use of angiotensin-converting enzyme inhibitors and AT1 receptor antagonists. These agents have been associated to antiproliferative, antifibrotic, and also antiinflammatory effects in the same model (Heemann and Lutz 2013; Szabo et al. 2000) as well as in humans.

However, trials combining angiotensin-converting enzyme inhibitors with AT1 receptor blockers were very disappointing so that the use of sequential blockade is not recommended at the moment for most patients (Persson and Rossing 2014).

Therefore, other therapeutic options would be very welcome for the treatment of patients with CKD. In this context, a combination with an antiinflammatory drug that does not have an influence on blood pressure might be a safe add-on as too aggressive blood-pressure lowering did have an adverse effect on hyperkalemia, acute kidney injury, and the incidence of major cardiovascular events (Fried et al. 2013). Lots of attention have recently been drawn to the new drug bardoxolone that activates nuclear 1 factor (erythroid-derived 2)-related factor 2 (Nrf2) transcription factor. The Nrf2 pathway is severely compromised in diabetic nephropathy and other entities of kidney disease and therefore activation was thought to slow the progression of kidney disease. Unfortunately, this was not the case and, even worse, there were more hospitalizations and deaths from cardiovascular events so that the trial was terminated before completion (de Zeeuw et al. 2013).

Is there evidence that blocking innate immunity might help to prevent acute or chronic kidney failure?

To start with, there is no published data on the use of any TLR antagonist for slowing CKD in patients. The TLR-4 receptor antagonist eritoran (E5564) has been used in the treatment of sepsis in humans with no effect on 28-day mortality (Opal et al. 2013). Even without being effective to treat septic patients, in this study, adverse events were comparable in the placebo and the treatment group and the drug was safe at least in the treatment window of 28 days.

Is there data from animal models that hint at a function of the TLR system in mediating CKD? The answer is plenty. Currently (July 2014), there are 429 citations for TLR and kidney mainly focusing on acute and chronic kidney injury in animal models. One of the crucial works linking innate immunity and acute kidney injury could show that TLR2deficient mice are protected from renal ischemia/reperfusion injury (I/R) (Leemans et al. 2005). This finding was confirmed for TLR 4 and double-deficient mice for TLR $2 / 4$ in the same model (Rusai et al. 2010). Moreover, the blocking of IL-1 RA was able to ameliorate kidney injury in a rat model of $I / R$ (Rusai et al. 2008).

An analogy to the 5/6 nephrectomy model is the model of chronic allograft damage in either rat or mice. In this model, TLR-deficient mice develop less inflammation, less infiltration of inflammatory cells, and most importantly better excretory renal function over time. Interestingly, the release of endogenous ligands in the kidney graft did not go down after the initial event of ischemia reperfusion but stayed rather high speaking for a self-sustaining cycle of TLR ligand release and TLR stimulation (Wang et al. 2010). Protective effects by deletion of TLR genes have also been demonstrated for the prevention of rejection in other organ systems.

To our knowledge, even in animal models apart from one work with eritoran for preventing $\mathrm{I} / \mathrm{R}$, no pharmacological intervention investigated the effects of a block of the TLR system in kidney disease (Liu et al. 2010). As promising as interfering with the TLR system might sound and as promising the results of experimental studies in animal models have been, to date, the data on use in humans is limited, not to say absent. One reason might be safety concerns as the TLR system is an essential part of every cell in the body. Permanently interfering with the TLR system which would be necessary in chronic kidney disease is a very promising but also very dangerous target as blockade might favor infections or trigger or sustain tumor growth. Data on downstream blockade of inflammation with tumor-necrosis-factor alpha 
blockers - a substance group which has been very well established in rheumatology and is considered a safe treatment option in this field - is very rare for treatment of chronic kidney disease (Venegas-Pont et al. 2010). In conclusion, the work of Kondo et al. (2014) is very important as it offers an alternative or add-on possibility to established medication interfering with the renin-angiotensin system. Before the substance can be used in human trials, considerable additional research is needed in animal models to prove that the substance is safe enough to go to phase I because the TLR system is such a mighty and potentially uncontrollable target.

\section{References}

de Zeeuw D, Akizawa T, Audhya P, Bakris GL, Chin M, Christ-Schmidt $\mathrm{H}$, Investigators BT (2013) Bardoxolone methyl in type 2 diabetes and stage 4 chronic kidney disease. N Engl J Med 369(26):24922503. doi:10.1056/NEJMoa1306033

Fried LF, Emanuele N, Zhang JH, Brophy M, Conner TA, Duckworth W, Investigators VN-D (2013) Combined angiotensin inhibition for the treatment of diabetic nephropathy. N Engl J Med 369(20):18921903. doi:10.1056/NEJMoa1303154

Heemann U, Lutz J (2013) Pathophysiology and treatment options of chronic renal allograft damage. Nephrol Dial Transplant 28(10): 2438-2446. doi:10.1093/ndt/gft087

Kliem V, Johnson RJ, Alpers CE, Yoshimura A, Couser WG, Koch KM, Floege J (1996) Mechanisms involved in the pathogenesis of tubulointerstitial fibrosis in 5/6-nephrectomized rats. Kidney Int 49(3):666-678

Kondo M., Tahara A., Hayashi K., Abe M., Inami H., Ishikawa T., Ito H., Tomura Y (2014) Renoprotective effects of novel interleukin-1 receptor-associated kinase 4 inhibitor AS2444697 through antiinflammatory action in 5/6 nephrectomized rats, Naunyn Schmiedebergs Arch Pharmacol., this issue
Leemans JC, Stokman G, Claessen N, Rouschop KM, Teske GJ, Kirschning CJ, Florquin S (2005) Renal-associated TLR2 mediates ischemia/reperfusion injury in the kidney. J Clin Invest 115(10): 2894-2903. doi:10.1172/JCI22832

Leemans JC, Kors L, Anders HJ, Florquin S (2014) Pattern recognition receptors and the inflammasome in kidney disease. Nat Rev Nephrol 10(7):398-414. doi:10.1038/nrneph.2014.91

Liu M, Gu M, Xu D, Lv Q, Zhang W, Wu Y (2010) Protective effects of Toll-like receptor 4 inhibitor eritoran on renal ischemia-reperfusion injury. Transplant Proc 42(5):1539-1544. doi:10.1016/j. transproceed.2010.03.133

Opal SM, Laterre PF, Francois B, LaRosa SP, Angus DC, Mira JP, Group, A. S (2013) Effect of eritoran, an antagonist of MD2-TLR4, on mortality in patients with severe sepsis: the ACCESS randomized trial. JAMA 309(11):1154-1162. doi:10.1001/jama.2013.2194

Persson F, Rossing P (2014) Sequential RAAS blockade: is it worth the risk? Adv Chron Kidney Dis 21(2):159-165. doi:10.1053/j.ackd. 2014.01.003

Rusai K, Huang H, Sayed N, Strobl M, Roos M, Schmaderer C, Lutz J (2008) Administration of interleukin-1 receptor antagonist ameliorates renal ischemia-reperfusion injury. Transpl Int 21(6):572-580. doi:10.1111/j.1432-2277.2008.00651.x

Rusai K, Sollinger D, Baumann M, Wagner B, Strobl M, Schmaderer C, Lutz J (2010) Toll-like receptors 2 and 4 in renal ischemia/ reperfusion injury. Pediatr Nephrol 25(5):853-860. doi:10.1007/ s00467-009-1422-4

Szabo A, Lutz J, Schleimer K, Antus B, Hamar P, Philipp T, Heemann U (2000) Effect of angiotensin-converting enzyme inhibition on growth factor mRNA in chronic renal allograft rejection in the rat. Kidney Int 57(3):982-991. doi:10.1046/j.1523-1755.2000.00926.x

Venegas-Pont M, Manigrasso MB, Grifoni SC, LaMarca BB, Maric C, Racusen LC, Ryan MJ (2010) Tumor necrosis factor-alpha antagonist etanercept decreases blood pressure and protects the kidney in a mouse model of systemic lupus erythematosus. Hypertension 56(4): 643-649. doi:10.1161/HYPERTENSIONAHA.110.157685

Wang Z, Wesche H, Stevens T, Walker N, Yeh WC (2009) IRAK-4 inhibitors for inflammation. Curr Top Med Chem 9(8):724-737

Wang S, Schmaderer C, Kiss E, Schmidt C, Bonrouhi M, Porubsky S, Grone HJ (2010) Recipient Toll-like receptors contribute to chronic graft dysfunction by both MyD88- and TRIF-dependent signaling. Dis Model Mech 3(1-2):92-103. doi:10.1242/dmm.003533 